\title{
LATTICE POINTS ON CIRCLES AND DISCRETE VELOCITY MODELS FOR THE BOLTZMANN EQUATION
}

\author{
LAURA FAINSILBER, PÄR KURLBERG, AND BERNT WENNBERG
}

\section{INTRODUCTION}

The phase space density $f$ of a dilute gas evolves according to the Boltzmann equation. In the physically relevant case, the gas would be confined to a subset $\Omega \subset \mathbb{R}^{3}$, and then $f(x, v, t): \Omega \times \mathbb{R}^{3} \times \mathbb{R}^{+} \rightarrow \mathbb{R}^{+}$, where $x$ denotes a position in space, $v \in \mathbb{R}^{3}$ is a velocity, and $t$ denotes the time. From a mathematical point of view, it is equally natural to consider the Boltzmann equation in any spatial dimension, and in some cases because of symmetries of $\Omega$, it is also relevant to consider $\Omega \subset \mathbb{R}^{d_{1}}$ and $v \in \mathbb{R}^{d_{2}}$ with $d_{1}<d_{2}$.

By a dilute gas we mean one where the particles interact with each other essentially only by pairwise interactions. Moreover, the Boltzmann equation assumes that the particles are so small compared to other distances, that they can be considered to be points.

Under these hypothesis, one can formally derive the Boltzmann equation (see [7])

$$
\partial_{t} f(x, v, t)+v \cdot \nabla_{x} f(x, v, t)=Q(f, f)(x, v, t) .
$$

The left hand side describes the evolution of the density by free transport, and the right hand side describes the impact of collisions. Per definition, a collision is a pairwise interaction that takes place instantaneously and at one single point in space. Hence $x$ and $t$ appear only as parameters in $Q(f, f)$, and we can write

$Q(f, f)(v)=\int_{\mathbb{R}^{d}} \int_{S^{d-1}}\left(f\left(v^{\prime}\right) f\left(v_{*}^{\prime}\right)-f(v) f\left(v_{*}\right)\right) q(|w|, \cos \theta) d S(u) d v_{*}$,

where the velocities "before and after a collision" are related by

$$
\begin{aligned}
& v^{\prime}=\frac{1}{2}\left(v+v_{*}\right)+|w| u \\
& v_{*}^{\prime}=\frac{1}{2}\left(v+v_{*}\right)-|w| u,
\end{aligned}
$$

We acknowledge partial support from the National Science Foundation (DMS 0071503) (P.K.), the Swedish Research Council (P.K. \& B.W.), the Royal Swedish Academy of Sciences (P.K.), and from the EC funded RTN network HYKE, Contract Number : HPRNCT-2002-00282 (B.W.) . 
with $w=\left(v_{*}-v\right) / 2$, and with $\cos \theta=\frac{u \cdot w}{|w|} ; d v_{*}$ is the Lebesgue measure in $\mathbb{R}^{d}$, and $d S(u)$ is the surface measure on $S^{d-1}$. Note that the pair of velocities before a collision, $v$ and $v_{*}$, and the pair of velocities after the collision, $v^{\prime}$ and $v_{*}^{\prime}$, are the endpoints of a diameter on the sphere which has its centre at $\frac{v+v_{*}}{2}$ and diameter $\left|v_{*}-v\right|$. This is exactly the condition needed in order that the collisions preserve the momentum and energy of the pair of particles. For $d=2$, the sphere becomes a circle, and this motivates the title of the paper.

In a discrete velocity model (DVM), the velocities are concentrated on a (usually finite) set of points $v_{j} \in \mathbb{R}^{d}$ in the velocity space:

$$
f(x, v, t)=\sum_{j} f_{j}(x, t) \delta_{v=v_{j}} .
$$

The Boltzmann equation (11) is then changed into a nonlinear system of conservation laws,

$$
\partial_{t} f_{j}+v_{j} \cdot \nabla_{x} f_{j}=\sum_{k, k^{\prime}, j^{\prime}} \Gamma_{j, k}^{j^{\prime}, k^{\prime}}\left(f_{j^{\prime}} f_{k^{\prime}}-f_{j} f_{k}\right)
$$

where the constants $\Gamma_{j, k}^{j^{\prime}, k^{\prime}} \geq 0$ must be chosen so that (4) makes sense from a physical point of view. In particular we require that $\left(v_{j}, v_{k}\right)$ and $\left(v_{j^{\prime}}, v_{k^{\prime}}\right)$ define two diameters on the same sphere, just as for the usual Boltzmann equation.

The first example of a discrete velocity model is that of Carleman ([4]), which has two velocities in $\mathbb{R}$. Many other models have been proposed, and there is a large literature on how to construct and analyse physically realistic models (i.e., that satisfy the right conservation laws and an entropy principle), see eg.[3].

Besides offering many interesting mathematical challenges (for example, there is no general theory of global existence of solutions to systems like (4)) the DVM:s are also candidates for the numerical approximation of the real Boltzmann equation (11). This leads naturally to the following question, which is the subject matter of the paper:

Suppose that we choose the discrete set of velocities to be $h \mathbb{Z}^{d}$, i.e. the integer lattice in $\mathbb{R}^{d}$, scaled by a factor $h$, and that we take

$$
f^{h}(v)=\sum_{\xi \in \mathbb{Z}^{d}} f_{\xi, h} \delta_{v=h \xi},
$$

so that $f^{h} \rightarrow f$, in some suitable sense, where $f \in L^{1}\left(\mathbb{R}^{d}\right)$. Is it then true that $Q\left(f^{h}, f^{h}\right)(v) \rightarrow Q(f, f)(v)$ for all $v \in h \mathbb{Z}^{d}$ when $h \rightarrow 0$ ?

This property, which is called consistency, together with stability are main ingredients when proving that a numerical method converges. 
The answer is yes. This was proven by Bobylev, Palczewski and Schnei$\operatorname{der}([2])$ for dimensions $d \geq 3$. In this paper, we prove that it is also true for $d=2$, and hence for all relevant cases.

Results of this kind are interesting, because they provide examples that are relevant to previous results of Desvillettes and Mischler ([9]), who proved that solutions to families of DVM:s can converge to DiPerna-Lions' solutions to (1) if certain conditions are satisfied.

Our result should not, however, be considered as relevant for numerical analysis, because the rate of convergence is so slow that a numerical method based on the theory presented here would hardly ever become useful.

The family of models considered here can be seen as coming from a rather straightforward discretization of the collision integral (2). This integral should be interpreted as an average over the $2 d-1$-dimensional manifold defined by

$$
\begin{aligned}
\mathcal{M}_{v}=\left\{\left(v_{*}, v^{\prime}, v_{*}^{\prime}\right) \in \mathbb{R}^{3 d}\right. & \text { s.t. } v^{\prime}+v_{*}^{\prime}-v_{*}=v \\
& \left.\left|v^{\prime}\right|^{2}+\left|v_{*}^{\prime}\right|^{2}-\left|v_{*}\right|^{2}=|v|^{2}\right\},
\end{aligned}
$$

and (2) is an iterated integral over this manifold. For a fixed $v$, we write $w=\left(v_{*}-v\right) / 2$, and then (3) becomes

$$
\begin{aligned}
v^{\prime} & =v+w+|w| u \\
v_{*}^{\prime} & =v+w-|w| u
\end{aligned}
$$

and also $v_{*}=v+2 w$. We then write

$$
g_{v}(w, u)=\left(f\left(v^{\prime}\right) f\left(v_{*}^{\prime}\right)-f(v) f\left(v_{*}\right)\right) q(|w|, \cos \theta),
$$

and so (after changing variables in the integral),

$$
Q(f, f)(v)=2^{d} \int_{\mathbb{R}^{d}}\left(\int_{S^{d-1}} g_{v}(w, u) d S(u)\right) d w .
$$

If $g$ is sufficiently regular (continuous), and decays sufficiently rapidly for large $w$, then the Riemann sum for the outer integral converges:

$$
\begin{aligned}
&(2 h)^{d} \sum_{\zeta \in \mathbb{Z}^{d}} \int_{S^{d-1}} g_{v}(h \zeta, u) d S(u) \\
& \longrightarrow 2^{d} \int_{\mathbb{R}^{N}}\left(\int_{S^{d-1}} g_{v}(w, u) d S(u)\right) d w
\end{aligned}
$$

when $h \rightarrow 0$. In order to construct a consistent DVM, it is then sufficient to evaluate the inner integral in terms of the values of $g$ on the lattice points $h \mathbb{Z}^{d}$, in such a way that the result converges to $\int_{S^{d-1}} g(w, u) d S(u)$. While with the formula (3), the collision integral should be taken over all $u \in$ 
$S^{d-1}$, we have here only access to those $u$ for which $v^{\prime}$ and $v_{*}^{\prime}$ belong to $h \mathbb{Z}^{d}$. But this is automatically achieved if $\zeta \in \mathbb{Z}^{d}$, and if $u=\zeta^{\prime} /\left|\zeta^{\prime}\right|$, where $\zeta^{\prime} \in \mathbb{Z}^{d}$ and $\left|\zeta^{\prime}\right|=|\zeta| ;$ then for all $v \in h \mathbb{Z}^{d}$,

$$
v+h \zeta \pm h|\zeta| u \in h \mathbb{Z}^{d} .
$$

However, note that with this construction, the center of the sphere is restricted to lie on a lattice point, and so it excludes cases like $v=(0,0)$, $v_{*}=(h, h)$.

Giving all points on the sphere equal weight, one arrives at the expression

$$
\frac{1}{r_{d}\left(|\zeta|^{2}\right)} \sum_{\substack{\zeta^{\prime} \in \mathbb{Z}^{d} \\\left|\zeta^{\prime}\right|=|\zeta|}}\left(f\left(v^{\prime}\right) f\left(v_{*}^{\prime}\right)-f(v) f\left(v_{*}\right)\right) q(|h \zeta|, \cos \theta)
$$

for approximating the inner integral in (7). The function $r_{d}(n)$ denotes the number of points with integer coordinates on a sphere in $\mathbb{R}^{d}$ with center at the origin and radius $\sqrt{n}$, i.e. the number of integer solutions to $x_{1}^{2}+\cdots+$ $x_{d}^{2}=n$.

We write, for all $v \in h \mathbb{Z}^{d}$.

$$
Q^{h}(f, f)(v)=
$$

$$
(2 h)^{d} \sum_{\zeta \in \mathbb{Z}^{d}} \frac{1}{r_{d}\left(|\zeta|^{2}\right)} \sum_{\substack{\zeta^{\prime} \in \mathbb{Z}^{d} \\\left|\zeta^{\prime}\right|=|\zeta|}}\left(f\left(v^{\prime}\right) f\left(v_{*}^{\prime}\right)-f(v) f\left(v_{*}\right)\right) q(|h \zeta|, \cos \theta) .
$$

In the two-dimensional case, all the terms in the sum are $2 \pi$-periodic functions of $\theta$, and assuming sufficient regularity, they can be expressed as a convergent Fourier series. It is then natural to introduce the exponential sum

$$
S(n, k)=\sum_{u \in \mathbb{Z}^{2}:|u|^{2}=n} e^{i k \theta_{u}}
$$

where $\theta_{u}$ is defined by $u=|u| \cdot\left(\sin \theta_{u}, \cos \theta_{u}\right)$. We will see in Section 4 that to prove that (8) converges to the angular integral in (7), it is enough to prove that for $k \neq 0$, the terms $S(n, k)$ converge to zero sufficiently fast as $n \rightarrow \infty$. Similar exponential sums are relevant for any dimension, and the work of Bobylev et al. also involves such estimates.

Here the needed estimate is given as Proposition 6 in Section 3 Then in Section 4 we put the estimates togheter to a proof of the main result:

Theorem 1. Consider the Boltzmann equation in two dimensions. Assume that $f$ and $q$ are so smooth that the function $g_{v}(w, u)$ defined in (6) is a $C^{2}$-function. Then for all $v \in h \mathbb{Z}^{2}$

$$
\left|Q(f, f)(v)-Q^{h}(f, f)(v)\right| \rightarrow 0
$$


when $h \rightarrow 0$.

Section 5] finally, contains a numerical illustration on the distribution terms where the circles contain many points, and we indicate how the computational cost could be reduced without loosing accuracy (still without the intention of actually giving an effective algorithm).

A more general construction of discrete velocity models on scaled integer lattices $h \mathbb{Z}^{2}$ consists in finding sets of integer points on the manifold $\mathcal{M}$ defined in (5). In this way, mass and energy conservation are automatically satisfied, but one also needs to verify that these are the only conserved quantities. And finally, in order that the models converge to the continuous model when $h \rightarrow 0$, it is necessary that the integer points are more or less uniformly distributed on $\mathcal{M}$.

The models studied here are constructed by discretizing, one at a time, the iterated integrals (2). An alternative way of writing this integral was introduced by Carleman [4]. Using that $v^{\prime}-v$ and $v_{*}^{\prime}-v$ are orthogonal, one can write (here we specialize to $d=3$ )

$$
\begin{aligned}
& Q(f, f)(v)= \\
& \quad \int_{\mathbb{R}^{3}} \int_{E_{v, v^{\prime}}}\left(f\left(v^{\prime}\right) f\left(v_{*}^{\prime}\right)-f(v) f\left(v_{*}\right)\right) q(w, \cos \theta) \frac{1}{\left|v-v^{\prime}\right|^{2}} d E\left(v_{*}^{\prime}\right) d v^{\prime},
\end{aligned}
$$

where $E_{v, v^{\prime}}$ is the plane that contains $v$ and is orthogonal to $v^{\prime}-v$, and where $d E\left(v_{*}^{\prime}\right)$ is the Euclidean measure on this plane. Heintz and Panferov [18] have analysed a DVM based on this interated integral, and proved that the method is consistent with the continuous model. This is somehow easier, because on all planes, the integer points are uniformly distributed, and they are all found by solving linear Diophantine equations. However, the density of points depends strongly on $v^{\prime}-v$, and so it is far from trival to prove the consistency. And again, the two-dimensional situation is more difficult, and has not yet been studied.

Yet another approach was introduced by Rogier and Schneider [24], who used the theory of Farey series to discretize the angular variable in the collision integral.

\section{NUMBER THEORETIC BACKGROUND}

2.1. Points on spheres; Asymptotics. To prove that (8) converges to the correct limit when $h \rightarrow 0$, one has to study the set

$$
\left\{\zeta /|\zeta|: \zeta \in \mathbb{Z}^{d},|\zeta|^{2}=n\right\}
$$

and to show that the points of this set are sufficiently well distributed on $S^{d-1}$ when $n$ is large; it is here that the number theoretical issues enter the 
game. Indeed, we can view the set of points with integer coordinates on a sphere of squared radius $n$ centered at the origin,

$$
\left\{\left(x_{1}, \ldots x_{d}\right) \in \mathbb{Z}^{d}, \sum_{i=1}^{d} x_{i}^{2}=n\right\}
$$

as the solution set for a quadratic form, and use the theory of integral quadratic forms to get estimates on the number of points (see for instance [13]). The expected number of points with integer coordinates on a sphere clearly depends on the dimension $d$. The naive approach to find the order of magnitude for a given dimension is to use the volume of a ball, divided by the number of spheres contained in the ball. The volume of a ball of radius $\sqrt{n}$ grows as $n^{d / 2}$ whilst the number of spheres is $n$. For $d=2$, this leads us to expect a constant number of lattice points on circles, for $d=3$ a growth proportional to $\sqrt{n}$, etc. However, for small $d$ this approach is misleading; the growth is quite irregular, and depends on the divisor structure of $n$. For $d=2$, we will see below that only values of $n$ of the form $n=2^{s} q^{2} p_{1}^{\alpha_{1}} \ldots p_{r}^{\alpha_{r}}$, where $q$ is a product of primes of the form $4 k+3$ and the $p_{i}$ 's are primes of the form $4 k+1$ (see below), yield circles with lattice points, and thus most circles have no points at all. In fact, Landau proved in 1908 that the number of circles with at least one lattice point, of integer squared radius smaller than $x$, grows as $C x / \sqrt{\log x}$. Moreover, there are also infinite families of circles with very few lattice points; radii that are a power of 2 yield 4 points for instance, and radii that are the square root of a prime of the form $p=4 k+1$ yield exactly 8 points. On the other hand, the number of lattice points on a circle is not bounded, for instance a circle with $n=p_{1} \ldots p_{r}$ as above where all the $p_{i}$ are distinct from each other has $4 \cdot 2^{r}$ points.

In dimension 3, all values of $n$ not of the form $n=4^{s}(8 k+7)$ yield spheres containing points with integer coordinates. This still leave a fairly large number of spheres with no points on, but for our purposes this does not really matter, as such spheres do not appear in the summation formulas (there is no relevant value for $\zeta$.) Among the spheres with lattice points, multiplying the radius by a power of 2 does not increase the number of points, but if we correct for this fact, the ratio between the number of points and the naive estimate is bounded, up to constants only depending on $\epsilon$, from above by $n^{\epsilon}$, and below by $n^{-\epsilon}$ for all $\epsilon>0$ (see [13] Ch. 4 for exact formulas involving class numbers or $L$-series.)

The higher-dimensional cases behave in a somewhat more regular fashion. Lagrange proved that every positive integer can be written as the sum of four squares, and thus for dimension $d \geq 4$, every sphere whose squared radius is an integer, has lattice points. For $d=4$ the number of points still 
oscillates rather wildly, with spheres with radius a power of 2 having just 24 points, but for greater dimensions, the naive estimate gives the correct asymptotic growth of the number of points.

Getting circles (or spheres) with "sufficiently many" lattice points, however, is not quite enough for our purposes: we also need that the lattice points are sufficiently uniformly distributed when projected on the unit sphere. In dimensions 3 and higher, this follows from estimates on Fourier coefficients of modular forms. The case $d \geq 4$, with some restrictions on the set of numbers in which $n$ tends to infinity when $d=4$, is due to Pommerenke [23]. For $d=3$, Duke [11] and Golubeva-Fomenko [14] used Iwaniec's [19] estimates on Fourier coefficients of half integral weight forms to obtain uniform distribution. Unfortunately, these techniques do not apply in dimension 2. Moreover, there are circles with large number of lattice points that are poorly distributed:

Theorem 2. (Cilleruelo [6]) For any $\epsilon>0$ and for any integer $k$, there exists a circle $x^{2}+y^{2}=n$ with more than $k$ lattice points such that all the lattice points are on the arcs $\sqrt{n} e^{(\pi / 2)(t+\theta) i}$ with $|\theta|<\epsilon, t \in\{0,1,2,3\}$.

On the other hand, we may use some other techniques from analytic number theory to show that lattice points on circles are equidistributed on average, and this is good enough for our purpose.

2.2. From points on circles to Gaussian integers. In the plane, we can view lattice points on a circle of radius $\sqrt{n}$, centered at the origin, as complex numbers with integer real and imaginary parts, and squared modulus $n$. It might seem as a trivial restatement, but doing so allows us to use use some techniques from algebraic number theory. The Gaussian integers, i.e., the set

$$
\mathbb{Z}[i]=\left\{x+i y \in \mathbb{C},(x, y) \in \mathbb{Z}^{2}\right\},
$$

is the ring of integers of the field $\mathbb{Q}(i)$. It shares an important property with the ordinary integers, namely unique factorization ${ }^{1}$, i.e., just as every integer in $\mathbb{Z}$ factors into prime numbers, and the factorization is unique up to ordering the primes and multiplying by -1 , Gaussian integers factor into Gaussian primes, uniquely up to ordering and multiplication by $-1, i,-i$ (these and 1 are the units, i.e. the elements having a multiplicative inverse in $\mathbb{Z}[i]$ ). For a more thorough introduction to primes in quadratic number fields, see for instance [17], Ch. XV.

The Gaussian primes (i.e. the elements of $\mathbb{Z}[i]$ that cannot be written as a product of Gaussian integers with smaller modulus), are of three types:

\footnotetext{
${ }^{1}$ This is rather unusual, the ring of integers in most number fields will not have this property.
} 
- the prime numbers $q \in \mathbb{Z}$ such that $q \equiv 3 \bmod 4$ remain prime in $\mathbb{Z}[i]$ (e.g. 3, $7,11,19, \ldots)$;

- for prime numbers $p \in \mathbb{Z}$ such that $p \equiv 1 \bmod 4$, there exists $x, y \in \mathbb{Z}$ s.t. $p=x^{2}+y^{2}$. Hence $p$ factors in $\mathbb{Z}[i]$ as a product of two Gaussian primes

$$
p=(x+i y)(x-i y)
$$

(e.g. 5 factors into $(2+i)(2-i)$ in $\mathbb{Z}[i])$

- last (and least!), $1+i$ is prime (note that $(1+i)(1-i)=2$ and that $1-i=-i(1+i)$ is merely "another form of the same prime" just as 3 and -3 represent the same prime).

If $n$ is the sum of two squares, then it can be factored in $\mathbb{Z}[i]$ :

$$
n=X^{2}+Y^{2}=(X+i Y)(X-i Y) .
$$

If $z=x+i y$ is a prime factor of $X+i Y$, then $\bar{z}=x-i y$ must be a prime factor of $X-i Y$. It follows that prime factors $q \equiv 3 \bmod 4$ of $n$ must appear in even powers. In addition, multiplying $n$ by an even power of a prime $q$ that is congruent with 3 mod 4 changes neither the number of solutions to $n=X^{2}+Y^{2}$ nor the distribution of arguments of the solutions.

Suppose now that $n$ contains a factor $p^{\alpha}$, where $p \equiv 1 \bmod 4$. The number $p$ can be factored in $\mathbb{Z}[i]$ as $(x+i y)(x-i y)$, and hence the multiplicity of $x+i y$ as a factor of $n$ is $\alpha$, and the same is true for $x-i y$. It follows that the multiplicity of $x+i y$ in $X+i Y$ can be any integer $j$, with $0 \leq j \leq \alpha$, and the multiplicty of $x-i y$ is then $\alpha-j$.

The same calculation can be done for powers of 2; however, the solutions given by different choices of $j$ in that case differ by a multiplication by a power of $i$, and so the power of 2 does not influence the number of solutions.

All solutions to $n=X^{2}+Y^{2}$ can now be expressed as $X+i Y=$ $\sqrt{n} \exp (i \theta)$, where all possible values of the argument $\theta$ can be computed as sums of terms deriving from the different factors of $n$ in the following way:

(1) $X+i Y$ can be multiplied by any unit, i.e. by \pm 1 or $\pm i$. This gives a term $k \pi / 2$ in the argument, $k=0,1,2,3$.

(2) If the multiplicity of 2 in $n$ is odd, then the argument must contain $\pi / 4$, the argument of $1+i$; the number of solutions does not change.

(3) For each prime factor $p \equiv 1 \bmod 4$ in $n$, let $\alpha_{p}$ be the multiplicity of $p$ in $n$, let $p=x_{p}^{2}+y_{p}^{2}$, and set $\theta_{p}=\arg \left(x_{p}+i y_{p}\right)$. For a particular choice of $j, 0 \leq j \leq \alpha_{p}$, the argument added to $X+i Y$ is $j \theta_{p}-\left(\alpha_{p}-j\right) \theta_{p}=\left(2 j-\alpha_{p}\right) \theta_{p}$. 
Since the choices of $k$, and of the different $j^{\prime} s$ are independent, the number of different solutions is $4 \prod_{p \equiv 1 \bmod 4}\left(\alpha_{p}+1\right)$.

2.3. Results on the distribution of primes and on the angular distribution of points. We will need the following results:

Theorem 3 (Merten's Theorem, see [17], Ch. 22.8).

$$
\prod_{\substack{p \leq x \\ \text { p prime }}}(1-1 / p) \sim e^{-\gamma / \log x}
$$

where $\gamma \simeq 0.57$ is Euler's constant.

As for the angular distribution of Gaussian primes, a result by Kubilyus gives that the angles $\left\{\theta_{p}\right\}_{p \equiv 1 \bmod 4}$ are equidistributed in $[0, \pi / 4]$ in the following sense:

Theorem 4 (Kubilyus, [21]). The number of Gaussian primes $\omega$ in the sector $0 \leq \alpha \leq \arg (\omega) \leq \beta \leq 2 \pi,|\omega|^{2} \leq u$ is equal to

$$
\frac{2}{\pi}(\beta-\alpha) \int_{2}^{u} \frac{d v}{\log v}+O(u \exp (-b \sqrt{\log u})),
$$

where $b$ is an absolute positive constant.

From Kubilyus' Theorem, it is straightforward to deduce (see [12], p. 92):

Corollary 5. If $k \in 4 \mathbb{N}$ and $\log k \leq b \sqrt{\log x}$, then

$$
\sum_{p \equiv 1} \frac{\left|\cos \left(k \theta_{p}\right)\right|}{p} \leq \frac{1}{\pi} \log \log x+(1-2 / \pi) \log \log k+O(1) .
$$

\section{EQUIDISTRIBUTION OF LATTICE POINTS ON CIRCLES}

What is needed for the proof of consistence of the discrete velocity model are estimates on the equidistribution of lattice points on circles.

The aim of this section is to show that lattice points on circles are equidistributed on average in the sense that the exponential sums $S(m, k)$ converge to zero when $m$ goes to infinity. We recall the definition of $S(m, k)$ :

$$
S(m, k)=\sum_{\left|w^{\prime}\right|^{2}=m} e^{i k \theta_{w^{\prime}}}
$$


Proposition 6. If $4 \nmid k$ then $|S(m, k)|=0$. If $4 \mid k$ and $k \neq 0$, there exist $C$ and $b>0$ such that

$$
\log \left(\frac{1}{X} \sum_{m \leq X}|S(m, k)|\right) \leq C-(1-2 / \pi) \log \left(\frac{\log X}{(\log |k|)^{2}}\right)
$$

for $X$ sufficiently large and $\log |k| \leq b \sqrt{\log X}$.

Remark: The mean discrepancy of the angles of Gaussian integers were studied by Kátai and Környei in [20], and by Erdős and Hall in [12]. Our method is similar to theirs, except that they bound

$$
\frac{1}{X / \sqrt{\log X}} \sum_{m \leq X} \frac{|S(m, k)|}{r(m)}
$$

instead of

$$
\frac{1}{X} \sum_{m \leq X}|S(m, k)|
$$

The proof is based on the observation that $|S(m, k)| / 4$ is a multiplicative function, i.e. a function $f: \mathbb{N} \rightarrow \mathbb{C}$ such that $f(m n)=f(m) f(n)$ for all $m, n$ such that $(m, n)=1$. It turns out that the mean value of a multiplicative function, under fairly general circumstances, can be bounded in terms of an exponential of a sum over primes. To make the paper more self contained, we include a weak form of the Halberstam-Richert inequality (cf. [15]).

Theorem 7. Let $f$ be a nonnegative multiplicative function such that

$$
\sum_{n \leq x} f(n)=O(x)
$$

and $f\left(p^{k}\right)=O(k)$ for all primes $p$ and $k \geq 1$. Then there exists $C>0$ such that

$$
\frac{1}{X} \sum_{m \leq X} f(m) \leq C \cdot \exp \left(\sum_{p \leq X} \frac{f(p)-1}{p}\right)+O\left(\frac{1}{\log X}\right)
$$

for all sufficiently large $X$.

Proof. Following Wirsing [25], let

$$
F(t)=\sum_{n \leq t} f(n) .
$$

Then

$$
\int_{1}^{X} \frac{F(t)}{t} d t=F(X) \log X+O(1)-\sum_{n \leq X} f(n) \log n
$$


On the other hand, by assumption, we have $F(t)=O(t)$, thus

$$
\int_{1}^{X} \frac{F(t)}{t} d t=O(X)
$$

and hence

$$
F(X) \log X \leq O(1)+X+\sum_{n \leq X} f(n) \log n .
$$

Using $\log n=\sum_{d \mid n} \Lambda(d)$, where $\Lambda$ is the von Mangoldt function ${ }^{2}$ we have

$$
\begin{gathered}
\sum_{n \leq X} f(n) \log n=\sum_{n \leq X} f(n) \sum_{d \mid n} \Lambda(d)=\sum_{d \leq X} \Lambda(d) \sum_{m \leq X / d} f(d m) \\
=\sum_{d \leq X} \Lambda(d) \sum_{\substack{m \leq X / d,(m, d)=1}} f(d m)+\sum_{d \leq X} \Lambda(d) \sum_{\substack{m \leq X / d,(m, d)>1}} f(d m) .
\end{gathered}
$$

Now, since $\Lambda(d)=0$ unless $d$ is a prime power, we have

$$
\begin{aligned}
\sum_{d \leq X} \Lambda(d) \sum_{\substack{m \leq X / d,(m, d)>1}} f(d m)= & \sum_{\substack{p^{k+l} \leq X \\
k, l \geq 1}} \log (p) \sum_{\substack{m \leq X / p^{k+l} \\
(p, m)=1}} f\left(p^{k+l} m\right) \\
& =\sum_{\substack{k+l \leq X \\
p^{k+l} \leq X}} \log (p) f\left(p^{k+l}\right) \sum_{\substack{m \leq X / p^{k+l} \\
k, l \geq 1}} f(m) .
\end{aligned}
$$

By the assumptions on $f$,

$$
f\left(p^{k+l}\right) \sum_{\substack{m \leq X / p^{k+l} \\(p, m)=1}} f(m) \leq O(k+l) \sum_{m \leq X / p^{k+l}} f(m)=O\left((k+l) \frac{X}{p^{k+l}}\right),
$$

and thus the second term in (12) is

$$
=O\left(\sum_{\substack{p^{n} \leq X \\ n \geq 2}} \log (p) n^{2} \frac{X}{p^{n}}\right)=O(X),
$$

since

$$
\sum_{p} \sum_{n \geq 2} \log (p) n^{2} p^{-n} \leq \sum_{p} \frac{\log (p)}{p^{2}} \sum_{m \geq 0}(2+m)^{2} 2^{-m}<\infty .
$$

\footnotetext{
${ }^{2}$ That is, $\Lambda(d)=\log p$ if $d=p^{k}$ and $k \geq 1$, otherwise $\Lambda(d)=0$.
} 
As for the first term in (12), we have (recall that $f$ is multiplicative and nonnegative)

$$
\begin{gathered}
\sum_{d \leq X} \Lambda(d) \sum_{\substack{m \leq X / d,(m, d)=1}} f(d m)=\sum_{d \leq X} \Lambda(d) f(d) \sum_{\substack{m \leq X / d \\
(m, d)=1}} f(m) \\
\leq \sum_{m \leq X} f(m) \sum_{d \leq X / m} \Lambda(d) f(d)
\end{gathered}
$$

Now,

$$
\sum_{d \leq X / m} \Lambda(d) f(d)=\sum_{\substack{p^{k} \leq X / m \\ k \geq 1}} \log (p) f\left(p^{k}\right) \leq \sum_{\substack{p^{k} \leq X / m \\ k \geq 1}} \log (p) O(k)=O(X / m)
$$

since

$$
\sum_{p \leq X / m} \log (p)=O(X / m)
$$

by the Prime number theorem, and

$$
\sum_{\substack{k \leq X / m \\ p^{k} \geq 2}} k \log (p)=O\left((X / m)^{1 / 2} \log ^{3}(X / m)\right)=O(X / m) .
$$

Thus,

$$
\sum_{m \leq X} f(m) \sum_{d \leq X / m} \Lambda(d) f(d)=O\left(\sum_{m \leq X} f(m) \frac{X}{m}\right) .
$$

But since $f$ is nonnegative and multiplicative, we have

$$
\begin{gathered}
\sum_{m \leq X} \frac{f(m)}{m} \leq \prod_{p \leq X}\left(1+f(p) / p+f\left(p^{2}\right) / p^{2}+\ldots\right) \\
\leq \prod_{p \leq X}\left((1+f(p) / p) \cdot\left(1+f\left(p^{2}\right) / p^{2}+f\left(p^{3}\right) / p^{3}+\ldots\right)\right),
\end{gathered}
$$

and since

$$
\sum_{p \leq X}\left(f\left(p^{2}\right) / p^{2}+f\left(p^{3}\right) / p^{3}+\ldots\right) \leq \sum_{p} \sum_{k \geq 2} \frac{O(k)}{p^{k}}<\infty,
$$

we find that

$$
\sum_{m \leq X} \frac{f(m)}{m}=O\left(\prod_{p \leq X}(1+f(p) / p)\right)
$$

Thus,

$$
F(X) \log X=O\left(X+X \cdot \prod_{p \leq X}(1+f(p) / p)\right)
$$


hence

$$
\frac{F(X)}{X}=O\left(\frac{1}{\log X}+\frac{\prod_{p \leq X}(1+f(p) / p)}{\log X}\right) .
$$

Now, by Merten's theorem, we have

$$
\prod_{p \leq X}(1-1 / p) \sim \frac{e^{-\gamma}}{\log X}
$$

and thus

$$
\begin{aligned}
\frac{F(X)}{X} & =O\left(\frac{1}{\log X}+\prod_{p \leq X}\left(1+\frac{f(p)-1}{p}-\frac{f(p)}{p^{2}}\right)\right) \\
& =O\left(\frac{1}{\log X}+\exp \left(\sum_{p \leq X} \frac{f(p)-1}{p}\right)\right) .
\end{aligned}
$$

Proof of Proposition 6 To see that $|S(m, k) / 4|$ is a multiplicative function, it is enough to recall the factorization of $m$ into Gaussian primes. Namely, if $p_{1}^{\alpha_{1}}, \ldots p_{J}^{\alpha_{J}}$ are all prime factors of $m$ with $p \equiv 1 \bmod 4$,

$$
S(m, k)=\sum_{\ell=0}^{3} i^{k \ell} \sum_{j_{1}=1}^{\alpha_{1}} \cdots \sum_{j_{J}=1}^{\alpha_{J}} e^{i k\left(\theta_{0}+\left(\alpha_{1}-2 j_{1}\right) \theta_{p_{1}}+\ldots+\left(\alpha_{J}-2 j_{J}\right) \theta_{p_{J}}\right)} .
$$

Here $\theta_{0}$ is a multiple of $\pi / 4$ which comes from powers of 2 in $m$, and the $\theta_{p_{j}}$ can be computed from the Gaussian factorization as described in Section 2.2 Also, because $\sum_{\ell=0}^{3} i^{k \ell}=4$ if $4 \mid k$ and zero otherwise,

$$
\frac{|S(m, k)|}{4}=\left|\sum_{j_{1}=1}^{\alpha_{1}} \cdots \sum_{j_{J}=1}^{\alpha_{J}} e^{i k\left(\left(\alpha_{1}-2 j_{1}\right) \theta_{p_{1}}+\ldots+\left(\alpha_{J}-2 j_{J}\right) \theta_{p_{J}}\right)}\right|,
$$

and this sum clearly factorizes, each factor containing a sum of terms corresponding to one of the prime factors $p$. Hence

$$
f_{k}(m)=\frac{|S(m, k)|}{4}
$$

is a nonnegative multiplicative function, as stated. In addition it satisfies $f_{k}(m) \leq r(m) / 4$ for all $m$. Thus, since

$$
\sum_{n \leq T} r(n)=\left|\left\{x, y \in \mathbb{Z}: x^{2}+y^{2} \leq T\right\}\right| \sim \pi(\sqrt{T})^{2}=\pi T
$$

we have

$$
\sum_{n \leq T} f_{k}(n)=O(T)
$$


Moreover, if $p \equiv 3 \bmod 4$ then

$$
f_{k}\left(p^{l}\right)=\left\{\begin{array}{l}
1 \text { if } l \text { is even, } \\
0 \text { if } l \text { is odd }
\end{array}\right.
$$

and if $p \equiv 1 \bmod 4$ then

$$
f_{k}\left(p^{l}\right)=\left|\sum_{j=0}^{l} e^{i k(l-2 j) \theta_{p}}\right|
$$

and thus $f_{k}\left(p^{l}\right) \leq l+1$ for all prime $p$ and $l \geq 1$. The assumptions in in Theorem 7 are thus satisfied, and we obtain

$$
\frac{1}{X} \sum_{m \leq X}|S(m, k)|=\frac{4}{X} \sum_{m \leq X} f_{k}(m) \leq C \exp \left(\sum_{p \leq X} \frac{f_{k}(p)-1}{p}\right)+O\left(\frac{1}{\log X}\right) .
$$

Now, by (14) and (15), we have

$$
f_{k}(p)=\left\{\begin{array}{lll}
2\left|\cos \left(k \theta_{p}\right)\right| & \text { if } p \equiv 1 \quad \bmod 4 \\
0 & \text { if } p \equiv 3 \quad \bmod 4
\end{array}\right.
$$

Hence

$$
\sum_{p \leq X} \frac{f_{k}(p)-1}{p}=\sum_{\substack{p \leq X \\ p \equiv 1}} \frac{2\left|\cos \left(k \theta_{p}\right)\right|}{p}-\sum_{p \leq X} \frac{1}{p}
$$

By Corollary 5

$$
\sum_{\substack{p \leq X \\ p \equiv 1}} \frac{2\left|\cos \left(k \theta_{p}\right)\right|}{p} \leq \frac{2}{\pi} \log \log X+2(1-2 / \pi) \log \log k+O(1) .
$$

if $\log k \leq b \sqrt{\log X}$. By Merten's theorem,

$$
\sum_{p \leq X} \frac{1}{p}=\log \log X+O(1)
$$

and thus

$$
\sum_{p \leq X} \frac{f_{k}(p)-1}{p} \leq(2 / \pi-1) \log \log x+2(1-2 / \pi) \log \log k+O(1) .
$$




\section{Proof of Theorem 1}

Here we carry out the steps of the proof as indicated in the introduction. First recall that the collision operator can be written

$$
Q(f, f)(v)=4 \int_{\mathbb{R}^{2}}\left(\int_{-\pi}^{\pi} g_{v}(w, \theta) d \theta\right) d w
$$

where, if we identify $u \in S^{1}$ with $\theta \in[-\pi, \pi[$,

$$
g_{v}(w, \theta)=q(|w|, \cos (\theta))\left(f\left(v^{\prime}\right) f\left(v_{*}^{\prime}\right)-f(v) f\left(v_{*}\right)\right),
$$

and

$$
\begin{aligned}
& v^{\prime}=v+w+R_{\theta} w \\
& v_{*}^{\prime}=v+w-R_{\theta} w ;
\end{aligned}
$$

as before, $w=\left(v_{*}-v\right) / 2$, and $R_{\theta}$ denotes a rotation by an angle $\theta$. Writing the Boltzmann equation for two-dimensional velocities, of course we have already stepped away from the physically realistic case, but disregarding this, a common assumption on $q$ is that

$$
q(|w|, \cos (\theta))=q_{1}(|w|) q_{2}(\theta),
$$

where $q_{1}(|w|) \sim|w|^{\alpha}$ for some $\alpha \in[0,1]$, and where $q_{2}(\theta) \sim|\theta|^{-\gamma}$ for some $\gamma \in] 1,3[$. This corresponds to a molecular interaction by hard inverse power law forces. With the stronger assumption that $q_{1}$ is smooth and strictly positive, it is possible to prove that there is a smooth solution $f(v, t)$ to the Boltzmann equation (see [10]), and then this also gives some regularity to $g(w, \theta)$, in spite of the singularity of $q_{2}$.

However, much work on the Boltzmann equation has been done with the hypothesis that $q$ is bounded or continuous with respect to $\theta$. With that assumption, the solution $f(v, t)$ keeps exactly the regularity of the initial data.

Because of this, it is relevant to assume whatever regularity of the solutions that is needed for the computations. With the aim of making the calculations easy, Theorem 1 has been written with unnecessarily strong hypothesis.

To simplify notation a little, let

$$
G_{v}(w)=\int_{-\pi}^{\pi} g_{v}(w, \theta) d \theta
$$

in the continuous case, and for the discrete case (then we assume, of course, that $v \in h \mathbb{Z}^{2}$ )

$$
G_{v}^{h}(h \zeta)=\frac{1}{r\left(|\zeta|^{2}\right)} \sum_{\substack{\zeta^{\prime} \in \mathbb{Z}^{d} \\\left|\zeta^{\prime}\right|=|\zeta|}} g_{v}(h \zeta, \theta)
$$


where $\theta$ is the angle between $\zeta^{\prime}$ and $\zeta$. As before, $r\left(|\zeta|^{2}\right)$ denotes the number of integer points on a sphere with radius $|\zeta|$.

Let

$$
Z_{h, R}=\left\{z \in \mathbb{Z}^{2} \text { s.t. }|z| \leq R / h\right\}
$$

for some $R>0$ (this is the most natural example, but other choices might be more efficient, as we shall see later). We want to prove that

$$
Q(f, f)(v)-(2 h)^{2} \sum_{\zeta \in Z_{h, R}} G_{v}^{h}(h \zeta) \rightarrow 0
$$

when $h \rightarrow 0$, and also make as precise a statement as possible about the rate of convergence.

Theorem 8. Suppose that $g_{v}(w, \theta)$ in (16) satisfies

(1) $g_{v}(w, \theta)$ is a $C^{1}$-function w.r.t. $w$

(2) $g_{v}(w, \theta)$ is a $C^{2}$-function w.r.t. $\theta$

(3) $\left\|g_{v}(\cdot, \theta)\left(1+|\cdot|^{2}\right)\right\|_{L^{1}(d w)} \leq C$

(This holds e.g. if the function $f$ and the crossection $q$ are $C^{2}$.) For given $R>0$ and $h>0$, let $Z_{h, R}$ be as in (17). Then given $\varepsilon>0$ there are reals $R>0$ and $h>0$ such that

$$
\left|Q(f, f)(v)-(2 h)^{2} \sum_{\zeta \in Z_{h, R}} G_{v}^{h}(h \zeta)\right| \leq \varepsilon .
$$

Proof. We still consider $Q(f, f)$ as an iterated integral, and write (for $v \in$ $\left.h \mathbb{Z}^{2}\right)$

$$
\begin{aligned}
Q(f, f)(v)-(2 h)^{2} \sum_{\zeta \in Z_{h, R}} G_{v}^{h}(h \zeta) & \\
= & \int_{\mathbb{R}^{2}} G_{v}(w) d w-(2 h)^{2} \sum_{\zeta \in Z_{h, R}} G_{v}(h \zeta) \\
& +(2 h)^{2} \sum_{\zeta \in Z_{h, R}}\left(G_{v}(h \zeta)-G_{v}^{h}(h \zeta)\right) .
\end{aligned}
$$

From the third part of the hypothesis on $g$ (which is implied by a decay of $f(v)$ for large velocities), it follows that for all $R>0$,

$$
\int_{|w| \geq R} G_{v}(w) d w \leq \frac{C_{1}}{R^{2}} .
$$

Continuity of $G_{v}(w)$ would be enough to conclude that

$$
\left|\int_{|w|<R} G_{v}(w) d w-(2 h)^{2} \sum_{\zeta \in Z_{h, R}} G_{v}(h \zeta)\right| \rightarrow 0
$$


when $h \rightarrow 0$. The hypothesis on $g_{v}(w, \theta)$ implies that actually $G_{v}(\cdot) \in C^{1}$, and there is a constant $C_{2}$ such that the difference is smaller than

$$
C_{2} R^{2} h=C \max _{w, j}\left|\partial_{w_{j}} G_{v}(w)\right| R^{2} h .
$$

Next we turn to the difference $G_{v}(h \zeta)-G_{v}^{h}(h \zeta)$, i.e. of

$$
\frac{1}{2 \pi} \int_{-\pi}^{\pi} g_{v}(h \zeta, \theta) d \theta-\frac{1}{r\left(|\zeta|^{2}\right)} \sum_{\substack{\zeta^{\prime} \in \mathbb{Z}^{2} \\\left|\zeta^{\prime}\right|=|\zeta|}} g_{v}(h \zeta, \theta)
$$

(recall that in the second term, $\theta$ is the angle between $\zeta^{\prime}$ and $\zeta$ ). We first write the periodic function $g_{v}(h \zeta, \theta)$ as a Fourier series,

$$
g_{v}(h \zeta, \theta)=\sum_{k \in \mathbb{Z}} \hat{g}_{v}(\zeta, k) e^{i k \theta}
$$

where

$$
\hat{g}_{v}(\zeta, k)=\frac{1}{2 \pi} \int_{-\pi}^{\pi} g_{v}(h \zeta, \theta) e^{-i k \theta} d \theta
$$

The assumptions on $g$ imply the existence of a constant $C_{3}$ so that

$$
\left|\hat{g}_{v}(\zeta, k)\right| \leq \frac{C_{3}}{1+k^{2}}
$$

Then (22) becomes

$$
\hat{g}_{v}(\zeta, 0)-\frac{1}{r\left(|\zeta|^{2}\right)} \sum_{\substack{\zeta^{\prime} \in \mathbb{Z}^{2} \\\left|\zeta^{\prime}\right|=|\zeta|}} \hat{g}_{v}(\zeta, 0)+\frac{1}{r\left(|\zeta|^{2}\right)} \sum_{\substack{\zeta^{\prime} \in \mathbb{Z}^{2} \\\left|\zeta^{\prime}\right|=|\zeta|}} \sum_{k \neq 0} \hat{g}_{v}(\zeta, k) e^{i k \theta}
$$

where the first terms cancel out, and only last sum remains. We next split that sum into a part with $|k| \leq M$, and a remainder, which can be made small by choosing $M$ large, if $g$ is sufficiently smooth with respect to $\theta$. Using (23),

$$
\left|\frac{1}{r\left(|\zeta|^{2}\right)} \sum_{\substack{\zeta^{\prime} \in \mathbb{Z}^{2} \\\left|\zeta^{\prime}\right|=|\zeta|}} \sum_{|k| \geq M} \hat{g}_{v}(\zeta, k) e^{i k \theta}\right| \leq 2 \frac{C_{3}}{M}
$$

To find the contribution of this term to (19), we multiply by $(2 h)^{2}$ and sum over $\zeta \in Z_{h, R}$ to find a bound of the form

$$
\frac{R^{2} C_{4}}{M}
$$


For the remaining part, using (23) again, we find a bound of the form

$$
\left|\sum_{0<|k|<M} \frac{C_{3}}{1+k^{2}} \frac{1}{r\left(|\zeta|^{2}\right)} \sum_{\substack{\zeta^{\prime} \in \mathbb{Z}^{2} \\\left|\zeta^{\prime}\right|=|\zeta|}} e^{i k \theta}\right| \leq \max _{0<|k|<M}\left|\frac{S\left(|\zeta|^{2}, k\right)}{r\left(|\zeta|^{2}\right)}\right| \cdot \sum_{0<|k|<M} \frac{C_{3}}{1+k^{2}}
$$

Adding the error terms (20), (21), (24) and (25) gives

$$
\begin{aligned}
& \left|Q(f, f)(v)-Q^{h}\left(f^{h}, f^{h}\right)(v)\right| \\
& \leq \frac{C_{1}}{R^{2}}+C_{2} R^{2} h+\frac{R^{2} C_{4}}{M}+C_{3}(2 h)^{2} \max _{0<|k|<M} \sum_{\zeta \in Z_{h, R}}\left|\frac{S\left(|\zeta|^{2}, k\right)}{r\left(|\zeta|^{2}\right)}\right|
\end{aligned}
$$

In the sum on the right hand side,

$$
\sum_{\zeta \in Z_{h, R}}\left|\frac{S\left(|\zeta|^{2}, k\right)}{r\left(|\zeta|^{2}\right)}\right|=\sum_{n<(R / h)^{2}}|S(n, k)|,
$$

and this can be estimated by using Proposition 6 with $X=(R / h)^{2}$. To do this, we must require that

$$
R / h>\exp \left(\log (M)^{2} / b\right)
$$

for some positive constant $b$. Then there is a constant $C_{5}$ such that

$$
\sum_{n<(R / h)^{2}}|S(n, k)| \leq C_{5}\left(\left(\frac{R}{h}\right)^{2} \exp \left(-\left(1-\frac{2}{\pi}\right) \frac{\log \left((R / h)^{2}\right)}{(\log M)^{2}}\right) .\right.
$$

The last term in (26) will always be the dominating one, and at this point, it does not give much to try to optimise the choices of $R, M$ and $h$. Hence to achieve an error of magnitude $\varepsilon$ we

(1) take $R=\sqrt{4 C_{1} / \varepsilon}$,

(2) observe that we must have $h<\varepsilon /\left(4 R^{2} C_{2}\right)=\varepsilon^{2} /\left(4 C_{1} C_{2}\right)$,

(3) choose $M=4 R^{2} C_{4} / \varepsilon=64 C_{1} C_{4} / \varepsilon^{2}$.

With these choises of $R$ and $M$, the last term can then be bounded by

$$
4 C_{3} C_{5} \frac{4 C_{1}}{\varepsilon} \exp \left(-\left(1-\frac{2}{\pi}\right) \log \frac{\log \left(4 C_{1} /\left(\varepsilon h^{2}\right)\right)}{\left(\log \left(64 C_{1} C_{4} / \varepsilon^{2}\right)\right)^{2}}\right),
$$

which converges to zero when $h \rightarrow 0$, and so there is an $h$ so small that also the last term in (26) is smaller than $\varepsilon / 4$. We see that in order to achieve an error of maginitude $\varepsilon$, one must take $h$ very small: $h=o\left(\exp \left(-2(\log \varepsilon)^{2} \varepsilon^{-2 /\left(1-\frac{2}{\pi}\right)}\right)\right)$ (note that (27) is then satisfied). 


\section{SOME EXAMPLES AND REMARKS}

From a numerical point of view, the discretization discussed above would be far too costly: a discrete velocity model with $N$ velocities would at least correspond to a computational cost of $O(N)$ per time step, because one needs to compute a value for each velocity. When the collision term is computed by the sum (18), the cost is $O\left(N^{2}\right)$ times some logarithmic factor of $N$ (which comes from the summation over the points on the circles). And the calculation above showed that $N$ grows exponentially in terms of the accuracy, $N \sim \frac{1}{h}>>\exp \left(\varepsilon^{-c}\right)$ for some positive constant $c$.

However, rather than estimating the computational cost in terms of the number of discretization points used, it is more relevant to give the cost in terms of the desired accuracy, given that the discretization points are used in an optimal way. The discussion around (18) suggests that one can reduce the computational cost considerably without compromising the order of accuracy. The poor rate of convergence is due to the approximation of $G_{v}(w)$. Generalizing the formula (18) slightly, we can write

$$
\int_{\mathbb{R}^{2}} G_{v}(w) d w \sim \frac{1}{\rho_{h}} \sum_{\zeta \in Z_{h}} G_{v}(h \zeta)
$$

where $\rho_{h}$ is the local density of $Z_{h}$. For $Z_{h}=\left\{\zeta \in \mathbb{Z}^{2}\right.$ s.t. $\left.|h \zeta| \leq R\right\}$, one has $\rho_{h}=h^{-2}$. Of course, even more generally one could take a local density which is not constant.

The procedure for constructing a DVM would then be

- Choose a density $\rho_{h}$ so that the sum (29) is approximated to the desired order.

- Choose $h$ so small that there exist a set $Z_{h}$ with this density so that for all $\zeta \in Z_{h}$ the angular integral is well approximated by the sum.

For such a model, the computational cost for each velocity would be of the order $\varepsilon^{-3}$ (this estimate is based on the assumption that the cost of evalutating the angular integral is $\varepsilon^{-1}$, and that the number of velocities is $O\left(\varepsilon^{-2}\right)$; lower cost can be acheived if higher order formulas are used for approximating the integrals). The problem remains, that a very large number of velocities are needed, and hence the total computational cost is still excessive. A more challenging task would be to dilute not only the set $Z_{h}$, but to choose in a systematic way subsets $U_{h} \subset h \mathbb{Z}^{2}$ for the discrete velocity model, so that $Q(f, f)(v)$ would be well approximated for all $v \in$ $U_{h}$, and to do this in a way that does not require too large tables for storing all possible collisions.

In the last part of this paper, we wish to illustrate the distribution of good radii. We then consider $\zeta=\left(\zeta_{1}, \zeta_{2}\right) \in \mathbb{Z}^{2}$ s.t. $0 \leq \zeta_{i}(i=1,2) \quad|\zeta|<$ $20000\}$. This is an extremely large set of points, which corresponds to a 
huge number of velocities (the $O\left(N^{2}\right)$ factor would in this case be of the order $10^{17}$, which is of course absurd)

Among the circles with radii $|\zeta|$ in this set, the largest number of points on one circle, is 384. In Fig. 1, we show all points $\zeta=\left(\zeta_{1}, \zeta_{2}\right)$ with $0<\zeta_{i}<$ 2000 , such that the circle passing through $\zeta$ has more than 72 points. There are 36163 points in this set. This is a small fraction of the total number of integer points, but they are seemingly well distributed, except near the origin.

Figure 2 shows points in the range $10000 \leq \zeta_{i} \leq 12000$. Here the small dots denote points on circles having at least 72 points, and the larger dots denote points on circles with at least 192 points (there are 141562 and 1120 points respectively in these sets).

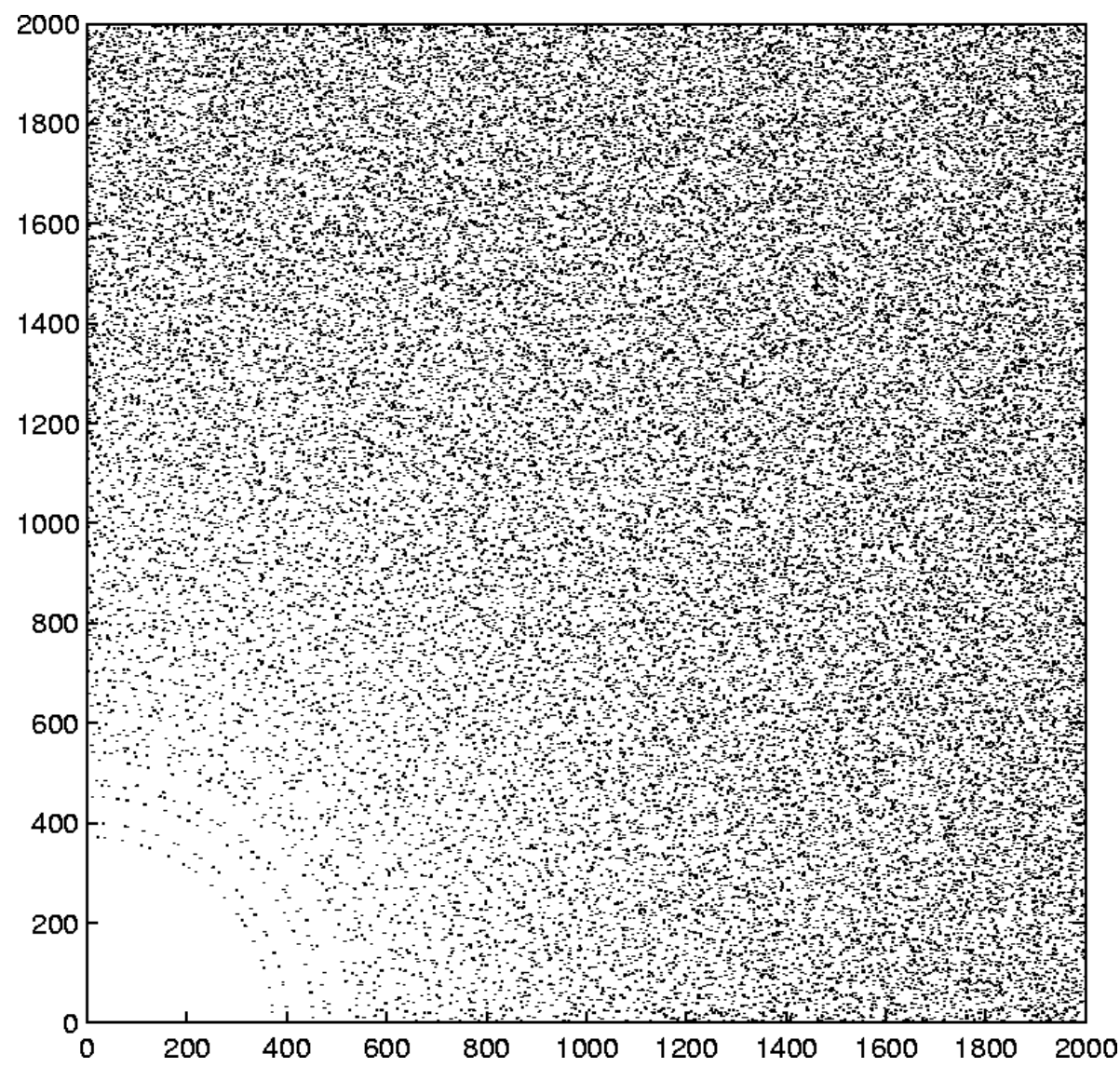

FIGURE 1. Lattice points such that circles containing these points, contain at least 72 lattice points

Acknowledgment: We would like to thank A. Bobylev, J. Brzezinski, A. Heintz, and Z. Rudnick for useful discussions. 


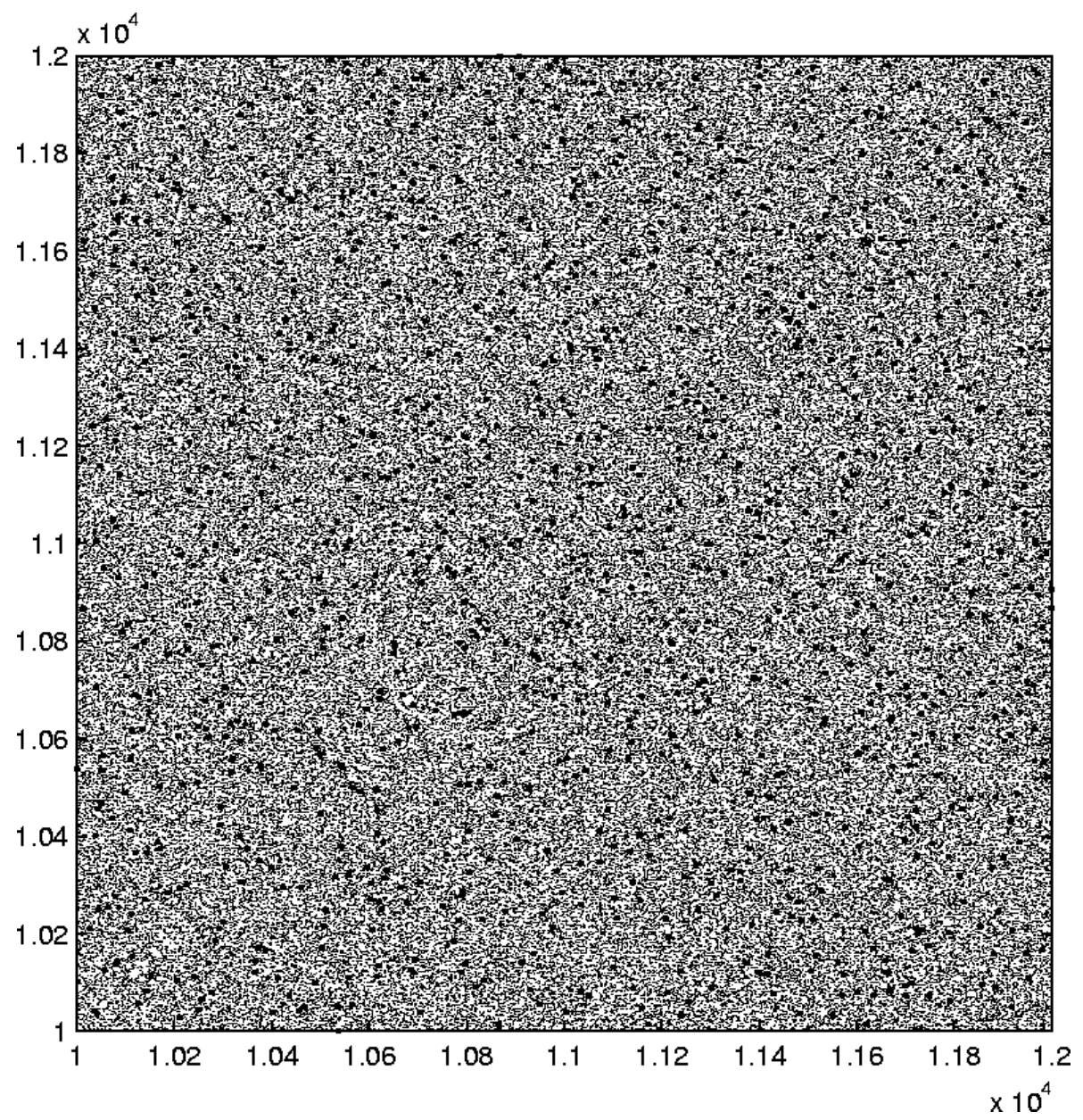

FIGURE 2. Lattice points such that circles containing these points, contain at least 72 lattice points (small dots), or at least 192 points (the larger dots)

\section{REFERENCES}

[1] A. V. Bobylev, A. Palczewski, and J. Schneider. On approximation of the Boltzmann equation by discrete velocity models. C. R. Acad. Sci. Paris Sér. I Math., 320(5):639644, 1995.

[2] A. Bobylev, A. Palczewski, J. Schneider: A consistency result for a discrete-velocity model of the Boltzmann equation. SIAM J. Numer. Anal. 34 (1997), no. 5, 1865-1883.

[3] A. Bobylev, C. Cercignani, Discrete velocity models without nonphysical invariants. J. Statist. Phys. 97 (1999),

[4] T. Carleman, Problèmes mathématiques dans la théorie cinétique des gaz, Almqvist \& Wiksell, Uppsala (1957).

[5] L. Carleson: Some analytic problems related to statistical mechanics, in Euclidean Harmonic Analysis J.J. Benedetto (ed.), LNM 779, Springer Verlag, Berlin Heidelberg, New York (1980). 
[6] J. Cilleruelo, The distribution of lattice points on circles, J. Number Theory 43 (1993), 198-202.

[7] C. Cercignani, R. Illner, M. Pulvirenti: The mathematical theory of dilute gases, Springer Verlag (1994).

[8] H. Davenport. Multiplicative number theory, volume 74 of Graduate Texts in Mathematics. Springer-Verlag, New York, third edition, 2000. Revised and with a preface by Hugh L. Montgomery.

[9] L. Desvillettes, S. Mischler: About the splitting algorithm for Boltzmann and B.G.K. equations. Math. Models Methods Appl. Sci. 6 (1996), no. 8, 1079-1101.

[10] L. Desvillettes, B. Wennberg: Regularity of solutions to the spatially homogeneous Boltzmann equation without cutoff, Comm. PDE 29 no $1 \& 2$ (2003), 133-156.

[11] W. Duke. Hyperbolic distribution problems and half-integral weight Maass forms. Invent. Math. 92 (1988), no. 1, 73-90.

[12] P. Erdős and R. R. Hall. On the angular distribution of Gaussian integers with fixed norm. Discrete Math., 200(1-3):87-94, 1999. Paul Erdős memorial collection.

[13] E. Grosswald, Representations of integers as sums of squares, Springer-Verlag, New York, 1985.

[14] E. P. Golubeva and O. M. Fomenko. Asymptotic distribution of lattice points on the three-dimensional sphere. Zap. Nauchn. Sem. Leningrad. Otdel. Mat. Inst. Steklov. (LOMI), 160 (1987), no. 8, 54-71, 297.

[15] H. Halberstam and H.-E. Richert. On a result of R. R. Hall. J. Number Theory, 11(1):76-89, 1979.

[16] R. R. Hall. A sharp inequality of Halász type for the mean value of a multiplicative arithmetic function. Mathematika, 42(1):144-157, 1995.

[17] G. H. Hardy and E. M. Wright. An introduction to the theory of numbers. Oxford, at the Clarendon Press, 1954. 3rd ed.

[18] V. Panferov, A. Heintz, A new consistent discrete-velocity model for the Boltzmann equation, Math. Methods Appl. Sci. 25 (2002), no. 7, 571-593.

[19] H. Iwaniec, Fourier coefficients of modular forms of half-integral weight, Invent. Math., 87 (1987), no. 2, 385-401.

[20] I. Kátai and I. Környei. On the distribution of lattice points on circles. Ann. Univ. Sci. Budapest. Eötvös Sect. Math., 19:87-91 (1977), 1976.

[21] I. Kubilyus. The distribution of Gaussian primes in sectors and contours. Leningrad. Gos. Univ. Uč. Zap. Ser. Mat. Nauk, 137(19):40-52, 1950.

[22] A. Palczewski, J. Schneider, Existence, stability, and convergence of solutions of discrete velocity models to the Boltzmann equation. J. Statist. Phys. 91 (1998), no. $1-2,307-326$.

[23] C. Pommerenke, Über die Gleichverteilung von Gitterpunkten auf $m$-dimensionalen Ellipsoiden, Acta Arith. 5 (1959), 227-257.

[24] F. Rogier, J. Schneider: A direct method for solving the Boltzmann equation, Transport Theory Statist. Phys. 23 (1994) 313-338

[25] E. Wirsing, Das asymptotische Verhalten von Summen über multiplikative Funktionen. Math. Ann. 143 (1961), 75-102. 
Department of Mathematics, Chalmers University of Technology, SE41296 Gothenburg, SwEDEN

E-mail address: laura@math. chalmers.se

E-mail address: kurlberg@math. chalmers.se

E-mail address: wennberg@math. chalmers.se 